\title{
Ectopic fat storage in heart, blood vessels and kidneys in the pathogenesis of cardiovascular diseases
}

\author{
Jean-Pierre Montani ${ }^{1}$, Joan F. Carroll ${ }^{2}$, Terry M. Dwyer ${ }^{3}$, Vladan Antic ${ }^{1}$, Zhihong \\ Yang ${ }^{1}$ and Abdul G. Dulloo ${ }^{1}$
}
${ }^{1}$ Department of Medicine/Division of Physiology, University of Fribourg, Switzerland
2 Department of Integrative Physiology, University of North Texas Health Science Center, Forth Worth, Texas, USA
${ }^{3}$ Department of Physiology and Biophysics, University of Mississippi Medical Center, Jackson, Mississippi, USA

Running head: Ectopic fat storage in key cardiovascular organs

Address for correspondence:

Jean-Pierre Montani

Department of Medicine/Division of Physiology

University of Fribourg

Rue du Musée 5

CH-1700 Switzerland

e-mail: jean-pierre.montani@unifr.ch 


\begin{abstract}
In humans and most animal models, the development of obesity leads not only to increased fat depots in classical adipose tissue locations, but also to significant lipid deposits within and around other tissues and organs, a phenomenon known as ectopic fat storage. The purpose of this review is to explore the possible locations of ectopic fat in key target-organs of cardiovascular control (heart, blood vessels and kidneys) and to propose how ectopic fat storage can play a role in the pathogenesis of cardiovascular diseases associated with obesity. In animals fed a high-fat diet, cardiac fat depots within and around the heart impair both systolic and diastolic functions, and may in the long term promote heart failure. Accumulation of fat around blood vessels (perivascular fat) may affect vascular function in a paracrine manner, as perivascular fat cells secrete vascular relaxing factors, pro-atherogenic cytokines and smooth muscle cell growth factors. Furthermore, high amounts of perivascular fat could mechanically contribute to the increased vascular stiffness seen in obesity. Finally, accumulation of fat in the renal sinus may limit the outflow of blood and lymph from the kidney, which would alter intrarenal physical forces and promote sodium reabsorption and arterial hypertension. Taken together, ectopic fat storage in key target-organs of cardiovascular control may impair their functions, contributing to the increased prevalence of cardiovascular diseases in obese subjects.
\end{abstract}

Keywords: Obesity, ectopic fat storage, lipotoxicity, perivascular fat, sinus lipomatosis, cardiovascular diseases, hypertension 


\section{Introduction}

In humans and most animal models, the development of obesity leads not only to increased fat depots in classical adipose tissue locations, but also to significant lipid deposits within and around other tissues and organs (ectopic fat storage). With fat gain, lipid deposition can impair tissue and organ function in two possible ways. First, the size of fat pads around key organs may increase substantially. This could modify organ function either by simple physical compression or because periorgan fat cells may secrete various locally acting substances. Second, lipid accumulation can occur in non-adipose cells and may lead to cell dysfunction or cell death, a phenomenon known as lipotoxicity. ${ }^{1,2}$ Intracellular lipid accumulations in endocrine pancreas, liver and skeletal muscle cells have all been described and contribute to the pathogenesis of impaired insulin secretion and insulin resistance, respectively. Ectopic fat deposition may also affect renal and cardiovascular function. The purpose of this review is to explore the possible locations of ectopic fat in key target-organs of cardiovascular control (heart, blood vessels and kidneys) and propose how ectopic fat storage can play a role in the pathogenesis of cardiovascular diseases.

In the last few years, ${ }^{3-8}$ a new animal model of obesity, the rabbit fed a high-fat diet has allowed us to study many of the mechanisms of obesity-associated cardiovascular pathologies. Ad libitum access to high-fat diet for 8-12 weeks led to a more than 3-fold increase in percent fat content determined by carcass analysis and lipid extraction, from $10 \%$ in lean rabbits to about $34 \%$ in obese rabbits. ${ }^{9}$ Fat depots occurred largely in the retroperitoneal space, in the neck, shoulder girdle and the subcutaneous suprapubic 
region. Smaller increases were seen in the mesentery, in the mediastinum, along blood vessels and in other scattered locations. Obese rabbits also gain lean mass. Indeed, as shown in Figure 1, all organs tested gained in weight with most of the gain due to an increase in lean mass (non-fat solids) plus the water obligated to that mass. There was considerable fat accretion in the renal sinus, the renal structure that starts from the hilum and intrudes upon the renal parenchyma.

Compared to lean rabbits, obese rabbits exhibit a higher 24-h blood pressure $(+15 \%)$ and heart rate $(+30 \%),{ }^{6}$ increased cardiac output, ${ }^{3}$ cardiac diastolic dysfunction ${ }^{5,10}$ and abnormal diurnal cardiovascular rhythms. ${ }^{8}$ Obese rabbits also exhibit neurohormonal changes with hyperinsulinemia, elevated plasma triglyceride levels and increased activity of the sympathetic nervous system and the renin-angiotensin system. ${ }^{4}$ Finally, obese rabbits show abnormal sodium handling with marked sodium retention during the development of obesity despite an elevated glomerular filtration rate. ${ }^{6,7}$ Similar findings are observed when dogs are fed a high-fat diet for several weeks. ${ }^{11,12}$ A model of obesity based solely on intake of a high-fat diet is relevant to the development of obesity in many situations in humans. Because obese dogs and rabbits exhibit many of the characteristics of obesity in humans, including hypertension, insulin resistance, humoral activation, increase in lean body mass and cardiac hypertrophy, they serve as a good model to study the cardiovascular effects of ectopic fat storage.

\section{Ectopic fat storage in the heart}

Most animal models of obesity as well as obese humans show an increased cardiac mass. In the rabbit model of obesity, a 50\% gain in body weight led to an increase in dry 
weight of the right and left ventricles by about $70 \%$ and $50 \%$, respectively, and to an increase in protein content by about $50 \%$ in both right and left ventricles. ${ }^{4}$ Since there were no significant alterations in total DNA content, these data suggest that weight gain causes cardiac myocyte hypertrophy. There was also a nearly 2 -fold increase of fat weight for both ventricles, which appears to be caused primarily by increased depots in the fat pads around the base of ventricles. Overall, those changes led to significant alterations in cardiac function. Study of the end-diastolic pressure-volume relationship in isolated hearts of lean and obese rabbits at different end-diastolic volumes shows a marked decrease in diastolic compliance. ${ }^{5}$ In itself, stiffer ventricles will impair cardiac function. A higher filling pressure is required to expand ventricles to ensure adequate filling and contraction. The concurrent increase in atrial pressures not only impairs venous return to the heart, but also increases intramyocardial capillary pressures and favors cardiac wall edema. ${ }^{13}$ In dogs, we have demonstrated that weight-gain with a high-fat diet for 4 weeks led to a significant increase in left atrial pressure, both at rest and more importantly during exercise, and to a net decrease in exercise tolerance. ${ }^{12}$ In addition, obese rabbit hearts exhibit mild systolic dysfunction with a reduced contractile responsiveness to isoproterenol. ${ }^{14}$

To what extent the cardiac changes observed in obesity are due to ectopic fat storage is not clear. Indeed, as illustrated in Figure 2, the causes of cardiac dysfunction in obesity are complex. It could be postulated that some of the cardiac changes result from the hypertension often observed in obesity. ${ }^{15}$ However, the increase in blood pressure is often very mild and is thus unlikely to cause by itself important structural cardiac changes. Furthermore, decrements in systolic and diastolic functions have been documented in obese subjects even in the absence of hypertension. ${ }^{16}$ Indeed, the Framingham study has determined that obesity is an independent risk factor for 
congestive heart failure. ${ }^{17}$ Another possible cause of cardiac dysfunction in obesity could be related to the consistent increase in cardiac output seen in obesity. The increase in both fat and organ masses raises whole body metabolic demands and thus regional blood flows. ${ }^{3}$ In turn, cardiac output, the sum of all regional blood flows, increases $(+37 \%$ in obese rabbits), putting an additional work load on the heart. Although experimental models of high-output heart failure, following the opening of an arteriovenous fistula for example, usually require larger increases in cardiac output (2-3 fold increase) for heart failure to develop, the contributing load of a moderately increased cardiac output, however, cannot be excluded. Indeed, the arteriovenous fistula of hemodialysis patients accounts for an about 30\% increase in cardiac output, and its closure in stable renal transplant patients can significantly decrease left ventricular mass. ${ }^{18}$ Finally, the combination of mild elevation of systolic blood pressure along with increased heart rate increases the double product (heart rate-pressure product), a determinant of myocardial oxygen consumption. ${ }^{19,20}$ Since weight gain leads to a reduced parasympathetic control of heart rate with a decreased baroreflex gain, ${ }^{21}$ small intermittent increases in blood pressure are not balanced by adequate decreases in heart rate and further increase heart work.

As shown in Figure 2, a contributory role of ectopic fat storage in the pathogenesis of cardiac dysfunction is suggested for the following reasons: (1) Accumulation of fat pads around ventricles (within a poorly distensible pericardium) may theoretically increase ventricular stiffness and thus contribute to diastolic dysfunction. To our knowledge, there are, however, no studies that have specifically addressed this point. (2) More importantly, weight gain may lead to intramyocytic deposits of lipids. Cardiac lipid accumulation has been observed in various rodent models of obesity. First, hearts from Zucker diabetic fatty (ZDF) fa/fa rats, a strain of rats characterized by leptin receptor 
deficiency, severe obesity and hyperinsulinemia, exhibit an elevation in myocardial triglyceride and ceramide contents that can be partially reversed by treatment with the antisteatotic drug troglitazone. ${ }^{22}$ ZDF rats have also a poor systolic function (as assessed by cardiac circumferential fractional shortening), which can be reversed by troglitazone treatment started early in life. It is postulated that the accumulation of large amounts of triglycerides may trigger a pathological signaling cascade resulting in apoptosis and systolic dysfunction. Second, hearts from obese, leptin-deficient, ob/ob mice showed marked accumulation of neutral lipid droplets within cardiac myocytes. ${ }^{23}$ In those mice, systolic function was only marginally affected, but there was a clear association between cardiac lipid accumulation and diastolic dysfunction. Finally, myocardial lipid content is also increased in lean Zucker rats fed a high-fat diet and is associated with myocardial oxidative injury. ${ }^{24}$ As for obese humans, lipid excess in the myocardium, as assessed by magnetic resonance spectroscopy, has been demonstrated and correlates with left ventricular mass and systolic dysfunction. ${ }^{25}$

In summary, genetic rodent models of obesity and diet-induced obesity in rodents and humans show cardiac lipid accumulation within myocytes. However, the quantitative contribution of cardiac lipotoxicity in the pathogenesis of cardiac dysfunction is not known. In fact, the combination of a mild increase in blood pressure, increase in cardiac output, increase in the heart rate-pressure product, and ectopic fat storage within and around the heart, could have additive effects resulting in the frequent observation of systolic and diastolic dysfunctions in obesity, which in the long term promote heart failure. In the follow-up of participants in the Framingham Heart Study, increased bodymass index was associated with an increased risk of heart failure. ${ }^{26}$ Given the high prevalence of obesity, it is not surprising that there is today a growing epidemic of heart failure. ${ }^{27}$ Furthermore, the poorer exercise tolerance in obesity, due to the combination of 
cardiac dysfunction, increased whole body metabolic demands and weight-induced osteoarticular problems, may further potentiate obesity, leading to a vicious cycle of inactivity, weight gain and heart failure. ${ }^{15}$

\section{Ectopic fat storage around blood vessels}

Nearly all blood vessels in the body are surrounded by variable amounts of adventitial fat. Traditionally, perivascular fat has been assumed to serve as structural support for the vasculature. However, perivascular fat has been shown to modulate vascular responsiveness to vasoactive agents in ex-vivo studies of the aorta ${ }^{28,29}$ and of the mesenteric vessels. ${ }^{30}$ Interestingly, diet-induced fat deposition in experimental models of obesity is not limited to the subcutaneous fat and to the classical visceral depots in tight connection with mediastinic and abdominal organs, but also occurs along the major blood vessels. ${ }^{9}$ In rats, high-fat feeding resulted in a 2 -fold increase in the mass of perivascular adipose tissue. $^{31}$ Obese Zucker $f a / f a$ rats have also significantly more periaortic fat than their lean littermates, even when periaortic fat is normalized to body weight. ${ }^{32}$

The pathophysiological consequences of increased perivascular fat in obesity are depicted in Figure 3. A first consequence could simply be mechanical. As more adipose tissue wraps around blood vessels, it is conceivable that the large amounts of perivascular fat could contribute to the increased vascular stiffness often observed in obese subjects. Although most vascular elasticity studies in humans have been conducted in small and medium size vessels accessible to ultrasonography, the vascular properties of large vessels in obese subjects have also been investigated. Excess body weight is associated

with higher aortic stiffness as determined by magnetic resonance imaging techniques ${ }^{33}$ or 
by aortic pulse wave velocity measurements. ${ }^{34}$ What is worrisome is that the strength of this association can be seen in very young adults. ${ }^{34}$ In fact, non-invasive measurements of the carotid artery by ultrasound imaging in severely obese children revealed a significantly greater carotid stiffness. ${ }^{35}$ Here again, it is not known to what extent perivascular fat contributes to the increased vascular stiffness. Other factors, such as obesity-induced hypertension, endothelial dysfunction, insulin resistance, higher sympathetic activity, the low-grade inflammation state of obesity ${ }^{36}$ and possibly lipid accumulation within vascular smooth muscle cells, ${ }^{37}$ may all contribute to the increased vascular stiffness of obesity. Whatever the cause, the loss of vascular distensibility has dramatic influences on cardiac function. A stiffer aorta per se increases pulse pressure simply because the aortic pressure changes created by ventricular ejection are no longer damped by an effective Windkessel function. A stiffer aorta also increases pulse wave velocity. Peripheral reflection waves return much quicker to the heart, increasing blood pressure during systole and no longer during diastole. As a consequence, there is a secondary elevation of systolic blood pressure (a phenomenon known as augmentation), which puts an additional work load on the heart. Furthermore, since the majority of left coronary blood flow occurs during diastole, the decrease in diastolic blood pressure may impair coronary blood flow reserve.

In addition, perivascular fat could act as secretory organ to influence the neighboring blood vessels. This function of perivascular fat is very intriguing since studies in isolated blood vessels have shown that the contractile response of rat aortic rings to angiotensin II, serotonin and phenylephrine was significantly attenuated in the presence of periadventitial fat. ${ }^{29}$ This effect is independent of nitric oxide formation and appears to be mediated by a transferable relaxing factor that is released by periadventitial vascular adipose tissue. Yet, it is not known whether this apparently beneficial effect of 
periadventitial fat could be impaired under certain pathological conditions. On the other hand, perivascular fat could have adverse effects, as shown on Figure 3, by releasing growth factors acting on the adjacent vascular smooth muscle cells (VSMC) or by secreting pro-atherogenic cytokines.

A plausible adipocyte-derived vascular growth factor is angiotensin II, a small peptide that has, among many other effects, vascular mitogenic and pro-inflammatory properties. ${ }^{38}$ Indeed, adipocytes can produce and release angiotensinogen and may have the complete enzymatic machinery for local production of angiotensin II. ${ }^{39}$ Furthermore, abdominal visceral fat has been shown to express more angiotensinogen than does subcutaneous fat. ${ }^{40}$ However, it is not known whether perivascular adipocytes can release angiotensinogen or angiotensin II in significant amounts to diffuse to and affect neighboring VSMC. Another growth factor candidate could be leptin, which has been shown to stimulate VSMC proliferation and migration. ${ }^{41}$ However, leptin expression is higher in subcutaneous fat than in visceral fat, and its effect on vascular growth may be mediated via circulating levels. In a study in 294 healthy adolescents with a broad range of body mass indexes, higher serum leptin concentrations were associated with impaired arterial distensibility, independently of fat mass or blood pressure. ${ }^{42}$ Intriguingly, leptindeficient $o b / o b$ mice or leptin-receptor deficient $d b / d b$ mice are relatively resistant to atherosclerosis despite being obese, hyperlipidemic and diabetic. ${ }^{43}$ Furthermore, carotid artery injury combined with cholesterol and high-fat diet in $o b / o b$ or $d b / d b$ mice failed to induce significant neointimal thickening whereas the same treatment was very effective in wild-type mice. ${ }^{44}$ To complicate the matter, obese subjects are known to develop leptin resistance; the role of leptin in promoting atherosclerosis in diet-induced obesity is therefore not clear. 
Other vascular growth factors could be involved. In cell culture experiments, adipose-conditioned medium stimulates the growth of human VSMC via a factor that is heat- and trypsin-resistant. ${ }^{32}$ Perivascular adipose-tissue conditioned medium is also capable of promoting VSMC growth. ${ }^{32}$ The growth-stimulating effect was more pronounced when using periaortic fat from older rats (24 months) than from younger rats (3 months). Therefore, adipocyte-derived VSMC growth factors could be involved in vascular remodeling, particularly in ageing. Furthermore, as this effect is dosedependent, higher amounts of perivascular fat could lead to more intense remodeling.

Alternatively, vascular growth could be promoted by a relative lack of growth inhibitors. For example, there is compelling evidence that adiponectin, a protein which is exclusively and abundantly produced by adipocytes, ${ }^{45}$ has a protective role in cardiovascular disease. ${ }^{46}$ In addition to its strong anti-inflammatory and anti-atherogenic properties, adiponectin suppresses VSMC proliferation and migration and could be an important modulator of vascular remodeling. ${ }^{47}$ Since obese subjects and patients with type 2 diabetes have low levels of plasma adiponectin, ${ }^{46}$ a postulated decreased secretion of adiponectin by perivascular adipocytes could promote vascular hypertrophy.

Finally, perivascular adipocytes could play a role in the progression of vascular dysfunction and atherosclerosis via secretion of various pro-inflammatory cytokines. Obesity is being increasingly recognized as a low-grade inflammatory state. ${ }^{36}$ Features of acute-phase activation and low-grade inflammation, including elevated circulating levels of fibrinogen, C-reactive protein (CRP), tumor necrosis factor- $\alpha$ (TNF- $\alpha$ ) and interleukin6 (IL-6) have been associated with (central) obesity. ${ }^{48}$ Positive association between body mass index and CRP has been described both in adults ${ }^{49}$ and children. ${ }^{50}$ The prevailing view is that inflammatory cytokines, which are released by adipose tissue, exert the proatherogenic effects via the systemic circulation by acting on leucocytes and endothelial 
cells. An alternate and emerging concept is that perivascular adipocytes could promote vascular dysfunction and atherosclerosis via a paracrine secretion of various proinflammatory cytokines, in particular certain pro-atherogenic chemokines (chemo(attractant cyto)kines). In fact, VSMC are potential targets for chemokines as they possess a number of functional chemokine receptors. ${ }^{51}$ In addition, aortic smooth muscle cells have been shown to produce CRP in response to inflammatory cytokines. ${ }^{52}$

Perivascular adipose tissue is tightly bound to the wall of large arteries, particularly around the aortic arch, the abdominal aorta and the iliac bifurcation, which are predilection sites for atherogenesis in humans. Since the adventitial tissue that separates perivascular fat from the media is usually relatively thin $(100-200 \mu \mathrm{m})$, it is postulated that chemokines produced by perivascular adipocytes might easily diffuse to the neighboring vascular media and thereby contribute to the progression of atherosclerosis. In general, adipose tissue has been shown to secrete various proatherogenic chemokines, such as interleukin-8 (IL-8) and monocyte chemoattractant protein-1 (MCP-1). In fact, visceral abdominal fat produces 2-3 fold more IL-8 than does subcutaneous abdominal fat. ${ }^{53}$ In addition, a recent study has reported that the proatherogenic chemokines RANTES (Regulated on Activated Normal T-cell Expressed and Secreted) and interferon- $\gamma$-inducible protein-10 (IP-10) can be produced by human white adipose tissue explants, and that expression of these two chemokines is upregulated in obese patients. $^{31}$ RANTES promotes plaque formation and antagonism of RANTES receptors reduces atherosclerotic plaque formation in low-density lipoprotein receptordeficient mice fed a high-cholesterol diet. ${ }^{54}$ IP-10 has mitogenic effects on VSMC and is a chemoattractant protein for lymphocytes and monocytes. ${ }^{55}$ A higher expression of IP10 in perivascular fat of obese patients could contribute to vascular stiffness and 
atherosclerosis. Whether perivascular fat can produce all these pro-inflammatory mediators awaits further exciting studies.

In summary, perivascular adipose tissue is in close connection with the media of blood vessels, separated from it only by a very thin adventitial layer. The mass of perivascular fat increases in obesity and it is postulated that a paracrine secretion of various growth-stimulating and pro-inflammatory agents could contribute to the pathogenesis of cardiovascular diseases.

\section{Ectopic fat storage in the kidney}

The development of obesity-induced hypertension in $\operatorname{dogs}^{11}$ and rabbits ${ }^{7}$ is associated with significant sodium retention in spite of a marked increase in glomerular filtration rate (GFR). Analysis of renal artery pressure-sodium excretion relationship in obese dogs fed a HFD for 5-6 weeks reveals a shift to the right as well as a decrease of the slope of the pressure-natriuresis curve. ${ }^{56}$ Many mechanisms (reviewed in Ref. ${ }^{15}$ ) have been suggested to explain this abnormal sodium handling, including increased renal nerve sympathetic activity, activation of the renin-angiotensin-aldosterone system, as well as direct sodium-retaining effects of insulin. In addition, there is evidence that weight gain is associated with abnormal physical forces within the kidney that could promote sodium retention. ${ }^{57}$ Measurement of renal interstitial fluid hydrostatic pressure (RIHP) in obese dogs show elevated values of about $19 \mathrm{mmHg}$ in comparison to values of 9-10 $\mathrm{mmHg}$ in lean dogs. Although small increases in RIHP tend to oppose tubular reabsorption forces, larger increases tend to compress medullary vasa recta and tubules, reducing vasa recta blood flow and tubular flow through the distensible loop of Henle. 
As postulated by Hall et al., ${ }^{57}$ the resulting increase in tubular transit time combined with the decrease in medullary blood flow promotes fluid and sodium reabsorption. In turn, this would decrease $\mathrm{NaCl}$ delivery to the macula densa, contributing to the increased GFR and renin secretion observed in obesity.

The mechanisms by which weight-gain leads to abnormal intrarenal physical forces are complex, but could be related to the marked structural renal changes observed in animal models of diet-induced obesity (dogs, rabbits). As shown in Figure 1, obese rabbits have larger kidneys $(+30 \%)$ and large fat deposits in the renal sinus. ${ }^{9}$ There was no detectable fat accumulation by extraction procedures within the renal parenchyma, but rather within the renal sinus. The renal sinus is the renal structure that starts from the hilum and intrudes upon the renal parenchyma, yet is physically separated from it by a reflection of the external capsule, ${ }^{58}$ as shown in the schematic diagram of Figure 4. Within this space, loose connective tissue surrounds renal blood and lymphatic vessels as well as the ureter.

As fat accretion fills the renal sinus, compression of various renal structures may occur. Compression of the inner medulla is likely since it is not protected by the renal fibrous capsule that corsets the entire kidney. Intriguingly, the content of interstitial hyaluronan, a space filling molecule normally found in the renal inner medulla, ${ }^{59}$ is markedly increased in obese $\operatorname{dogs}^{60}$ and rabbits. ${ }^{61}$ The expansion of the hyaluronan-filled extracellular matrix between vasa recta and thin Henle tubules, evidenced with PAS and Alcian blue staining on histological section of the medulla, ${ }^{61}$ may thus contribute to the compression of medullary structures. The mechanisms by which obesity results in increased intrarenal medullary hyaluronan are not known.

In addition, fat deposition within the renal sinus may compress the renal vein and renal lymphatic vessels, all easily compressible structures. Renal vein constriction results 
in increases in renal interstitial pressure. ${ }^{62}$ The increase in RIHP is much greater in situations of volume expansion and is then accompanied by a net decrease in fractional sodium excretion ${ }^{63}$ with a nearly doubling of the transit time through the loop of Henle. ${ }^{64}$ Since obesity is characterized by plasma volume expansion, one could speculate that the combination of slightly elevated vena caval pressure and renal vein constriction may contribute to the tendency for sodium retention during the development of obesity. In addition, renal lymphatic compression may be involved. Selective acute obstruction to renal lymphatic output increases kidney volume. ${ }^{65}$ Renal vein constriction increases kidney volume and renal lymph flow, ${ }^{65}$ which may serve to attenuate the increase in RIHP. Although renal lymph vessels and the renal vein are probably only mildly compressed by sinus lipomatosis, one cannot exclude additive effects and more serious increases in RIHP than would have been observed by constriction of each structure separately.

Furthermore, intra-abdominal pressure, which is directly related to the degree of abdominal adiposity, ${ }^{66}$ may amplify the compression due to renal sinus lipomatosis. In lean rabbits, the kidneys lay partially submersed in the ventral surface of the retroperitoneal fat. In obese rabbits, fat completely surrounds the kidneys, although well separated from the renal parenchyma by the fibrous capsule. Increased retroperitoneal fat and increased abdominal pressure could prevent the fat accreted in the renal sinus from expanding outwards and may thereby further restrict the renal outflow of urine, blood and lymph, thus elevating RIHP. 


\section{Summary and conclusions}

Obesity is one of the most serious health problems in industrialized societies. The increase in morbidity and mortality associated with weight gain results primarily from increased cardiovascular diseases such as stroke, coronary artery disease, congestive heart failure and chronic renal failure. Obesity-associated cardiovascular diseases have a complex, multifactorial origin. Arterial hypertension, which may be present in up to $50 \%$ of obese individuals, and metabolic problems with diabetes and dyslipidemia, whose prevalences increase dramatically with weight gain, are certainly major contributors. Accumulation of fat within and around key-organs of cardiovascular control may also affect their functions. Increased epicardial fat pads associated with intramyocardial lipid deposition may lead to both systolic and diastolic dysfunctions. Accumulation of fat within the renal sinus associated with the increased intra-abdominal pressure of visceral obesity may compress the renal papilla, the renal vein and lymphatics vessels, altering intrarenal physical forces that favor sodium reabsorption and arterial hypertension. Finally, very promising is the emerging concept that accumulation of fat around blood vessels (perivascular fat) may promote vascular remodeling and atherosclerosis via a paracrine secretion of smooth muscle cell growth regulators and pro-atherogenic cytokines. Taken together, ectopic fat storage in key target-organs of cardiovascular control may contribute to the increased prevalence of cardiovascular diseases in obese subjects. 


\section{References}

1. Unger RH, Orci L. Diseases of liporegulation: new perspective on obesity and related disorders. FASEB J 2001; 15: 312-21.

2. Schaffer JE. Lipotoxicity: when tissues overeat. Curr Opin Lipidol 2003; 14: 281-7.

3. Carroll JF, Huang M, Hester RL, Cockrell K, Mizelle HL. Hemodynamic alterations in hypertensive obese rabbits. Hypertension 1995; 26: 465-70.

4. Carroll JF, Dwyer TM, Grady AW, Reinhart GA, Montani JP, Cockrell K, Meydrech EF, Mizelle HL. Hypertension, cardiac hypertrophy, and neurohumoral activity in a new animal model of obesity. Am J Physiol 1996; 271: H373-H378.

5. Carroll JF, Summers RL, Dzielak DJ, Cockrell K, Montani JP, Mizelle HL. Diastolic compliance is reduced in obese rabbits. Hypertension 1999; 33: 811-5.

6. Antic V, Tempini A, Montani JP. Serial changes in cardiovascular and renal function of rabbits ingesting a high-fat, high-calorie diet. Am J Hypertens 1999; 12: 826-9.

7. Antic V, Kiener-Belforti F, Tempini A, Van Vliet BN, Montani JP. Role of the sympathetic nervous system during the development of obesity-induced hypertension in rabbits. Am J Hypertens 2000; 13: 556-9.

8. Antic V, Van Vliet BN, Montani JP. Loss of nocturnal dipping of blood pressure and heart rate in obesity-induced hypertension in rabbits. Auton Neurosci 2001; 90: $152-7$.

9. Dwyer TM, Mizelle HL, Cockrell K, Buhner P. Renal sinus lipomatosis and body composition in hypertensive, obese rabbits. Int J Obes Relat Metab Disord 1995; 19: $869-74$.

10. Carroll JF, Braden DS, Cockrell K, Mizelle HL. Obese hypertensive rabbits develop concentric and eccentric hypertrophy and diastolic filling abnormalities. Am J Hypertens 1997; 10: 230-3.

11. Hall JE, Brands MW, Dixon WN, Smith MJ, Jr. Obesity-induced hypertension. Renal function and systemic hemodynamics. Hypertension 1993; 22: 292-9.

12. Mizelle HL, Edwards TC, Montani JP. Abnormal cardiovascular responses to exercise during the development of obesity in dogs. Am J Hypertens 1994; 7: 374-8.

13. Laine GA, Allen SJ. Left ventricular myocardial edema. Lymph flow, interstitial fibrosis, and cardiac function. Circ Res 1991; 68: 1713-21.

14. Carroll JF, Jones AE, Hester RL, Reinhart GA, Cockrell K, Mizelle HL. Reduced cardiac contractile responsiveness to isoproterenol in obese rabbits. Hypertension 1997; 30: 1376-81. 
15. Montani JP, Antic V, Yang Z, Dulloo A. Pathways from obesity to hypertension: from the perspective of a vicious triangle. Int J Obes Relat Metab Disord 2002; 26 Suppl 2: S28-S38.

16. Scaglione R, Dichiara MA, Indovina A, Lipari R, Ganguzza A, Parrinello G, Capuana G, Merlino G, Licata G. Left ventricular diastolic and systolic function in normotensive obese subjects: influence of degree and duration of obesity. Eur Heart J 1992; 13: 738-42.

17. Hubert HB, Feinleib M, McNamara PM, Castelli WP. Obesity as an independent risk factor for cardiovascular disease: a 26-year follow-up of participants in the Framingham Heart Study. Circulation 1983; 67: 968-77.

18. van Duijnhoven EC, Cheriex EC, Tordoir JH, Kooman JP, van Hooff JP. Effect of closure of the arteriovenous fistula on left ventricular dimensions in renal transplant patients. Nephrol Dial Transplant 2001; 16: 368-72.

19. Van Vliet BN, Montani JP. Baroreflex stabilization of the double product. Am J Physiol 1999; 277: H1679-H1689.

20. Van Vliet BN, Belforti F, Montani JP. Baroreflex stabilization of the double (pressure-rate) product at $0.05 \mathrm{~Hz}$ in conscious rabbits. Am J Physiol Regul Integr Comp Physiol 2002; 282: R1746-R1753.

21. Van Vliet BN, Hall JE, Mizelle HL, Montani JP, Smith MJ, Jr. Reduced parasympathetic control of heart rate in obese dogs. Am J Physiol 1995; 269: H629H637.

22. Zhou YT, Grayburn P, Karim A, Shimabukuro M, Higa M, Baetens D, Orci L, Unger RH. Lipotoxic heart disease in obese rats: implications for human obesity. Proc Natl Acad Sci U S A 2000; 97: 1784-9.

23. Christoffersen C, Bollano E, Lindegaard ML, Bartels ED, Goetze JP, Andersen CB, Nielsen LB. Cardiac lipid accumulation associated with diastolic dysfunction in obese mice. Endocrinology 2003; 144: 3483-90.

24. Vincent HK, Powers SK, Dirks AJ, Scarpace PJ. Mechanism for obesity-induced increase in myocardial lipid peroxidation. Int J Obes Relat Metab Disord 2001; 25: 378-88.

25. Szczepaniak LS, Dobbins RL, Metzger GJ, Sartoni-D'Ambrosia G, Arbique D, Vongpatanasin W, Unger R, Victor RG. Myocardial triglycerides and systolic function in humans: in vivo evaluation by localized proton spectroscopy and cardiac imaging. Magn Reson Med 2003; 49: 417-23.

26. Kenchaiah S, Evans JC, Levy D, Wilson PW, Benjamin EJ, Larson MG, Kannel WB, Vasan RS. Obesity and the risk of heart failure. N Engl J Med 2002; 347: 30513. 
27. Fonarow GC, Horwich TB. Prevention of heart failure: effective strategies to combat the growing epidemic. Rev Cardiovasc Med 2003; 4: 8-17.

28. Soltis EE, Cassis LA. Influence of perivascular adipose tissue on rat aortic smooth muscle responsiveness. Clin Exp Hypertens A 1991; 13: 277-96.

29. Lohn M, Dubrovska G, Lauterbach B, Luft FC, Gollasch M, Sharma AM. Periadventitial fat releases a vascular relaxing factor. FASEB J 2002; 16: 1057-63.

30. Verlohren S, Dubrovska G, Tsang SY, Essin K, Luft FC, Huang Y, Gollasch M. Visceral Periadventitial Adipose Tissue Regulates Arterial Tone of Mesenteric Arteries. Hypertension 2004; 44: 271-6.

31. Henrichot E, Juge-Aubry CE, Wohlwend A, Somm E, Pernin A, Pauchot J, Giusti V, Dayer JM, Chizzolini C, Meier CA. Production of IP-10 and RANTES by human adipose tissue: A role for perivascular adipocytes in atherosclerosis? [Abstract]. Annual Meeting of the Endocrine Society 2004; OR-34-6.

32. Barandier $\mathrm{C}$, Yang Z. Adipocytes release a heat- and trypsin-resistant growth factor for vascular smooth muscle cells: effects of obesity and aging [Abstract]. European Society of Cardiology Congress 2004; P374.

33. Danias PG, Tritos NA, Stuber M, Botnar RM, Kissinger KV, Manning WJ. Comparison of aortic elasticity determined by cardiovascular magnetic resonance imaging in obese versus lean adults. Am J Cardiol 2003; 91: 195-9.

34. Wildman RP, Mackey RH, Bostom A, Thompson T, Sutton-Tyrrell K. Measures of obesity are associated with vascular stiffness in young and older adults. Hypertension 2003; 42: 468-73.

35. Tounian P, Aggoun Y, Dubern B, Varille V, Guy-Grand B, Sidi D, Girardet JP, Bonnet D. Presence of increased stiffness of the common carotid artery and endothelial dysfunction in severely obese children: a prospective study. Lancet 2001; 358: 1400-4.

36. Yudkin JS. Adipose tissue, insulin action and vascular disease: inflammatory signals. Int J Obes Relat Metab Disord 2003; 27 Suppl 3: S25-S28.

37. Tertov VV, Orekhov AN, Martsenyuk ON, Perova NV, Smirnov VN. Low-density lipoproteins isolated from the blood of patients with coronary heart disease induce the accumulation of lipids in human aortic cells. Exp Mol Pathol 1989; 50: 337-47.

38. Dzau VJ. Theodore Cooper Lecture: Tissue angiotensin and pathobiology of vascular disease: a unifying hypothesis. Hypertension 2001; 37: 1047-52.

39. Engeli S, Schling P, Gorzelniak K, Boschmann M, Janke J, Ailhaud G, Teboul M, Massiera F, Sharma AM. The adipose-tissue renin-angiotensin-aldosterone system: role in the metabolic syndrome? Int J Biochem Cell Biol 2003; 35: 807-25. 
40. Giacchetti G, Faloia E, Mariniello B, Sardu C, Gatti C, Camilloni MA, Guerrieri M, Mantero F. Overexpression of the renin-angiotensin system in human visceral adipose tissue in normal and overweight subjects. Am J Hypertens 2002; 15: 381-8.

41. Oda A, Taniguchi T, Yokoyama M. Leptin stimulates rat aortic smooth muscle cell proliferation and migration. Kobe J Med Sci 2001; 47: 141-50.

42. Singhal A, Farooqi IS, Cole TJ, O'Rahilly S, Fewtrell M, Kattenhorn M, Lucas A, Deanfield J. Influence of leptin on arterial distensibility: a novel link between obesity and cardiovascular disease? Circulation 2002; 106: 1919-24.

43. Nishina PM, Naggert JK, Verstuyft J, Paigen B. Atherosclerosis in genetically obese mice: the mutants obese, diabetes, fat, tubby, and lethal yellow. Metabolism 1994; 43: $554-8$.

44. Schafer K, Halle M, Goeschen C, Dellas C, Pynn M, Loskutoff DJ, Konstantinides $\mathrm{S}$. Leptin promotes vascular remodeling and neointimal growth in mice. Arterioscler Thromb Vasc Biol 2004; 24: 112-7.

45. Scherer PE, Williams S, Fogliano M, Baldini G, Lodish HF. A novel serum protein similar to C1q, produced exclusively in adipocytes. J Biol Chem 1995; 270: 267469.

46. Ouchi N, Kihara S, Funahashi T, Matsuzawa Y, Walsh K. Obesity, adiponectin and vascular inflammatory disease. Curr Opin Lipidol 2003; 14: 561-6.

47. Arita Y, Kihara S, Ouchi N, Maeda K, Kuriyama H, Okamoto Y, Kumada M, Hotta K, Nishida M, Takahashi M, Nakamura T, Shimomura I, Muraguchi M, Ohmoto Y, Funahashi T, Matsuzawa Y. Adipocyte-derived plasma protein adiponectin acts as a platelet-derived growth factor-BB-binding protein and regulates growth factorinduced common postreceptor signal in vascular smooth muscle cell. Circulation 2002; 105: 2893-8.

48. Yudkin JS, Stehouwer CD, Emeis JJ, Coppack SW. C-reactive protein in healthy subjects: associations with obesity, insulin resistance, and endothelial dysfunction: a potential role for cytokines originating from adipose tissue? Arterioscler Thromb Vasc Biol 1999; 19: 972-8.

49. Visser M, Bouter LM, McQuillan GM, Wener MH, Harris TB. Elevated C-reactive protein levels in overweight and obese adults. JAMA 1999; 282: 2131-5.

50. Visser M, Bouter LM, McQuillan GM, Wener MH, Harris TB. Low-grade systemic inflammation in overweight children. Pediatrics 2001; 107: E13.

51. Schecter AD, Berman AB, Taubman MB. Chemokine receptors in vascular smooth muscle. Microcirculation 2003; 10: 265-72.

52. Calabro P, Willerson JT, Yeh ET. Inflammatory cytokines stimulated C-reactive protein production by human coronary artery smooth muscle cells. Circulation 2003; 108: 1930-2. 
53. Bruun JM, Lihn AS, Madan AK, Pedersen SB, Schiott KM, Fain JN, Richelsen B. Higher production of IL-8 in visceral vs. subcutaneous adipose tissue. Implication of nonadipose cells in adipose tissue. Am J Physiol Endocrinol Metab 2004; 286: E8-13.

54. Veillard NR, Kwak B, Pelli G, Mulhaupt F, James RW, Proudfoot AE, Mach F. Antagonism of RANTES receptors reduces atherosclerotic plaque formation in mice. Circ Res 2004; 94: 253-61.

55. Gerszten RE, Mach F, Sauty A, Rosenzweig A, Luster AD. Chemokines, leukocytes, and atherosclerosis. J Lab Clin Med 2000; 136: 87-92.

56. Granger JP, West D, Scott J. Abnormal pressure natriuresis in the dog model of obesity-induced hypertension. Hypertension 1994; 23: I8-11.

57. Hall JE. Mechanisms of abnormal renal sodium handling in obesity hypertension. Am J Hypertens 1997; 10: 49S-55S.

58. Kaissling B, Kriz W. Structural analysis of the rabbit kidney. Adv Anat Embryol Cell Biol 1979; 56: 1-123.

59. Knepper MA, Saidel GM, Hascall VC, Dwyer T. Concentration of solutes in the renal inner medulla: interstitial hyaluronan as a mechano-osmotic transducer. Am J Physiol Renal Physiol 2003; 284: F433-F446.

60. Alonso-Galicia M, Dwyer TM, Herrera GA, Hall JE. Increased hyaluronic acid in the inner renal medulla of obese dogs. Hypertension 1995; 25: 888-92.

61. Dwyer TM, Banks SA, Alonso-Galicia M, Cockrell K, Carroll JF, Bigler SA, Hall JE. Distribution of renal medullary hyaluronan in lean and obese rabbits. Kidney Int 2000; 58: 721-9.

62. Ott CE, Navar LG, Guyton AC. Pressures in static and dynamic states from capsules implanted in the kidney. Am J Physiol 1971; 221: 394-400.

63. Burnett JC, Jr., Knox FG. Renal interstitial pressure and sodium excretion during renal vein constriction. Am J Physiol 1980; 238: F279-F282.

64. Burnett JC, Jr., Haas JA, Knox FG. Segmental analysis of sodium reabsorption during renal vein constriction. Am J Physiol 1982; 243: F19-F22.

65. Stolarczyk J, Carone FA. Effects of renal lymphatic occlusion and venous constriction on renal function. Am J Pathol 1975; 78: 285-96.

66. Sugerman H, Windsor A, Bessos M, Wolfe L. Intra-abdominal pressure, sagittal abdominal diameter and obesity comorbidity. J Intern Med 1997; 241: 71-9. 


\section{Figure legends}

Fig 1 Obesity-related gain in the mass of organ/tissues in rabbits fed a high-fat diet. The bar chart represents the percent increase in total mass for each organ or tissue studied. Each bar is further broken according to chemical composition: tissue water, defined as the weight lost after drying; fat, defined as the weight loss after extraction by organic solvents; and lean mass, which is the weight after drying and organic extraction.

Fig 2 Postulated mechanisms by which diet-induced obesity may contribute to cardiac dysfunction. Hemodynamic changes with increases in blood pressure (BP), cardiac output (CO) and double product (DP) play an important role, but ectopic fat both around the ventricles and within cardiomyocytes probably worsens cardiac function.

Fig 3 Postulated mechanisms by which increased perivascular adipose tissue during weight gain may contribute to the development, maintenance or aggravation of cardiovascular diseases.

Fig 4 Schematic diagram of a unipapillate kidney typical of a rabbit, rat or mouse, illustrating how accumulation of fat in the renal sinus (sinus lipomatosis) may favor compression of the renal inner medulla and of hilum structures. 


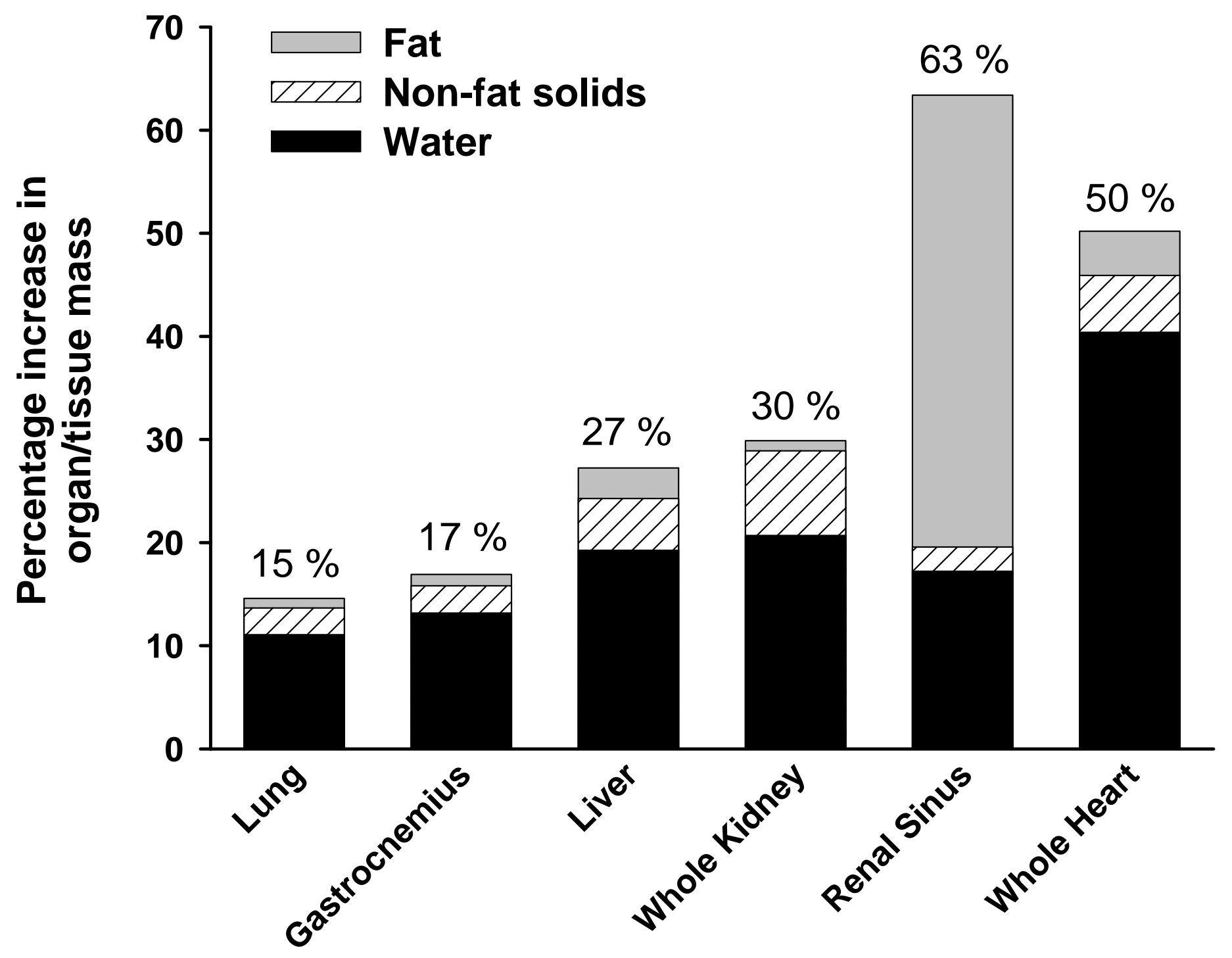




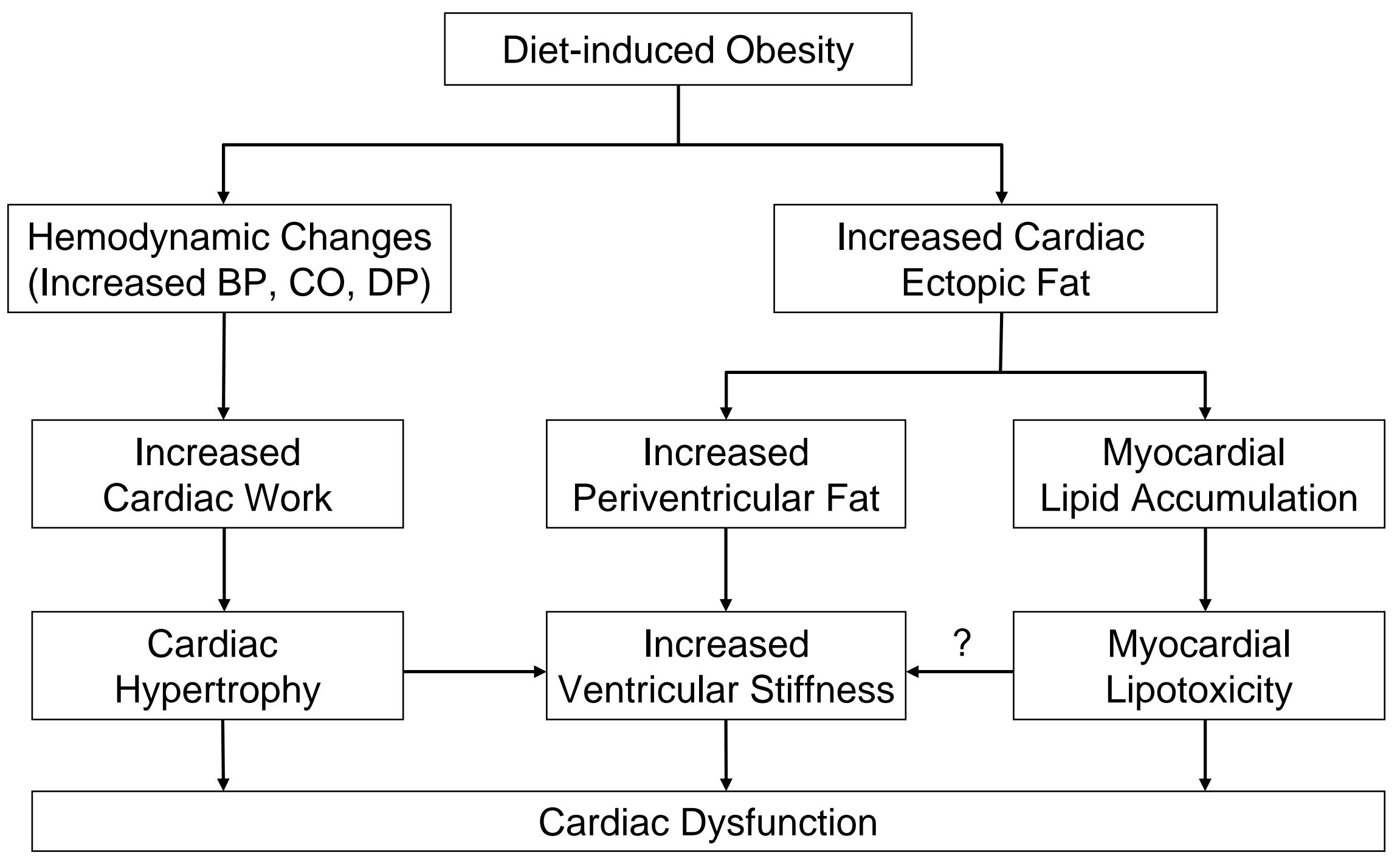




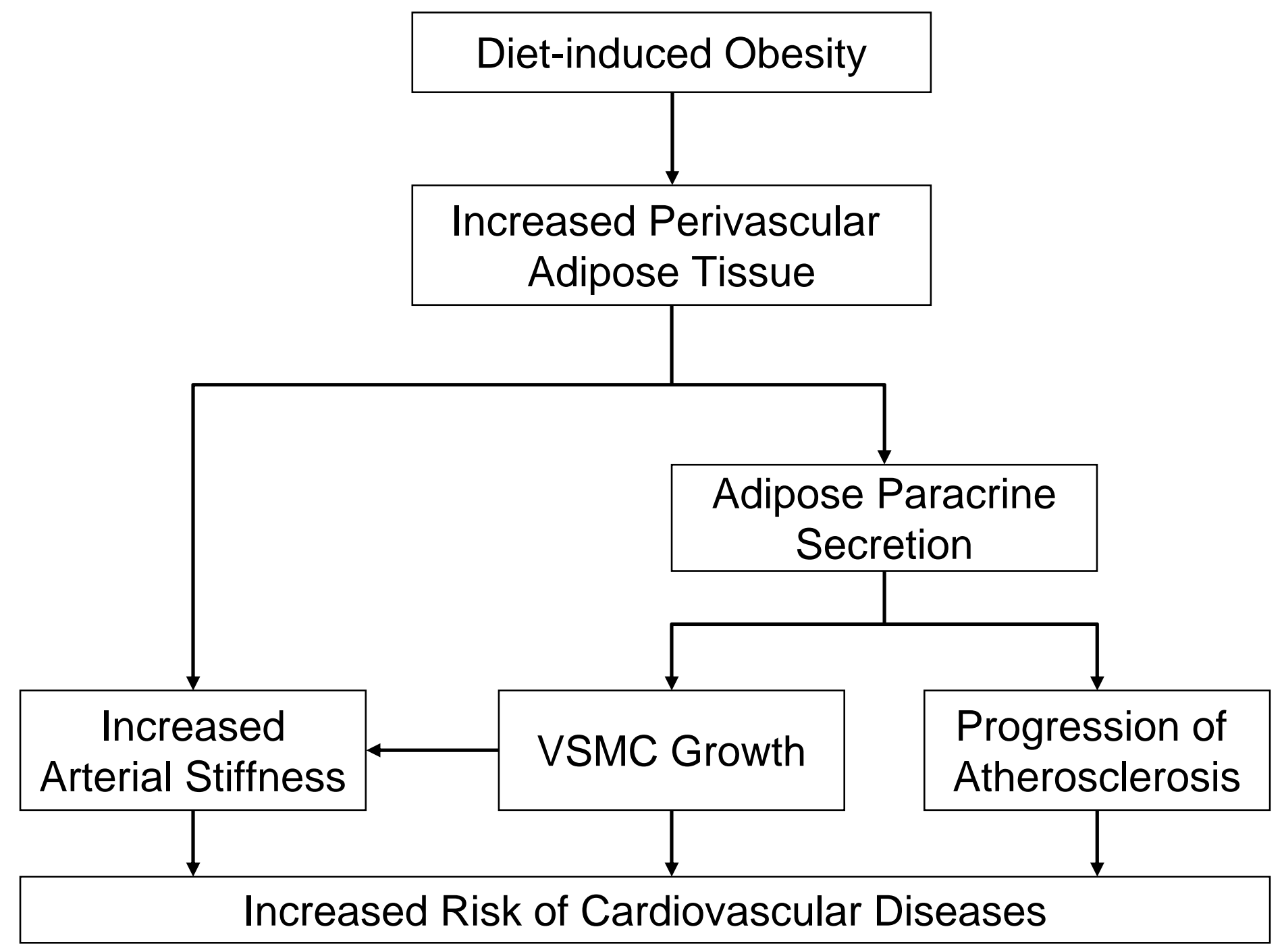




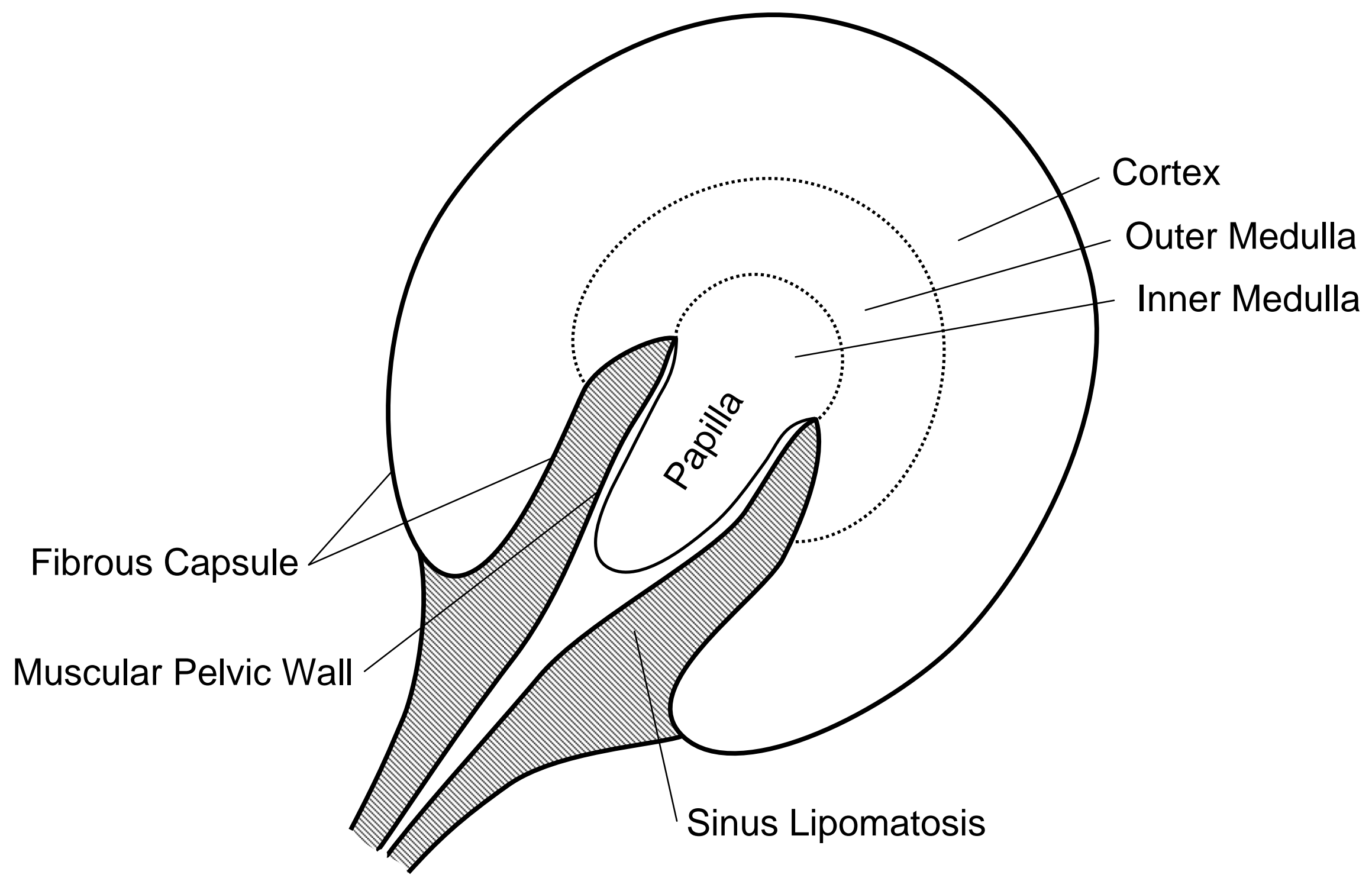

